\title{
Blazar monitoring with LOFAR
}

\section{Sara Turriziani ${ }^{1}$, Martin Hardcastle ${ }^{2}$, James Miller-Jones ${ }^{3}$, Jess Broderick $^{4}$ and Sera Markoff ${ }^{5}$ (on behalf of the Transients Key Science Project)}

\author{
${ }^{1}$ Dip. Fisica, Università degli studi di Roma "Tor Vergata" \\ via della Ricerca Scientifica 1, 00133 Roma, Italy \\ email: sara.turriziani@roma2.infn.it \\ ${ }^{2}$ School of Physics, Astronomy and Mathematics, University of Hertfordshire \\ College Lane, Hatfield AL10 9AB, UK email: m.j.hardcastle@herts.ac.uk \\ ${ }^{3}$ International Centre for Radio Astronomy Research, Curtin University \\ GPO Box U1987, Perth, WA 6845, Australia \\ email: james.miller-jones@curtin.edu.au \\ ${ }^{4}$ Astrophysics, Department of Physics, Oxford University \\ Keble Road, Oxford OX1 3RH \\ email: Jess.Broderick@astro.ox.ac.uk \\ 5 Astronomical Institute "Anton Pannekoek", University of Amsterdam \\ Science Park 904, 1098XH, Amsterdam, the Netherlands \\ email: s.b.markoff@uva.nl
}

\begin{abstract}
We have been monitoring some well-known bright blazars with short LOFAR observations since February 2013 with fortnightly cadence using the full available bandwidth of the High Band Antennas (i.e., $48 \mathrm{MHz}$ centred at $226 \mathrm{MHz}$ ). The sources were chosen to be bright at low frequencies and to exhibit strong $\mathrm{GHz}$-frequency radio variability on timescales of weeks to years. None of the five objects selected have been monitored previously in the $\mathrm{MHz}$ band. Here we report some preliminary results on flux variability obtained so far with LOFAR. These observations are scientifically valuable in their own right and also act as a proof of principle for broader, higher-cadence monitoring of the extragalactic sky with LOFAR and possibly SKA.
\end{abstract}

Keywords. galaxies: active, galaxies: jets, radio continuum: galaxies, techniques: interferometric

\section{Introduction}

The LOw Frequency ARray (LOFAR, van Haarlem et al. (2013)) is a network of radio telescopes that is centered in the Netherlands, but with international stations distributed across Europe, located in Germany, Sweden, the UK and France. It started as a new and innovative effort to force a breakthrough in sensitivity for astronomical observations at radio-frequencies below $250 \mathrm{MHz}$. Thanks to its high sensitivity and superb angular resolution, it allows us to systematically image and monitor blazars at the lowest available frequencies (corresponding to the lowest-energy electrons accessible to us) for the first time. Time variability is in fact the most powerful key we have now to understand these puzzling sources, in particular regarding the measurement of the physical parameters of the emission region, which can in turn put unique constraints on emission models and also on the poorly-understood low energy end of the particle energy spectrum.

\section{LOFAR blazar monitoring}

Refractive interstellar scintillation (RISS), caused by scattering in the turbulent ISM of our Galaxy, is commonly invoked to explain the low frequency variability of quasars 
Table 1. LOFAR Blazar Monitoring: the targets

\begin{tabular}{|c|c|c|c|c|c|c|c|c|}
\hline Source & & $\mathrm{R}$. & & & Dec & $\begin{array}{c}\text { Estimated Flux }{ }^{a} \\
\left(\mathrm{Jy} \mathrm{bm}^{-1}\right)\end{array}$ & $\begin{array}{l}\text { LOFAR Flux } \\
\quad\left(\mathrm{Jy} \mathrm{bm}^{-1}\right)\end{array}$ & $\begin{array}{l}\text { Light curve } \\
\text { (any trend?) }\end{array}$ \\
\hline AO $0235+164$ & $02^{h}$ & $38^{m}$ & $38.930^{s}$ & $16^{d}$ & $36^{\prime} 59.275^{\prime \prime}$ & - & $\sim 0.8$ & no \\
\hline S5 $0716+714$ & $07^{h}$ & $21^{m}$ & $53.448^{s}$ & $71^{d}$ & $20 ' 36.363 "$ & 1.6 & $\sim 1.9$ & no \\
\hline Mrk 501 & $16^{h}$ & $53^{m}$ & $52.217^{s}$ & $39^{d}$ & $45^{\prime} 36.609 "$ & 1.9 & $\sim 1.6$ & yes \\
\hline BL Lac & $22^{h}$ & $02^{m}$ & $43.291^{s}$ & $42^{d}$ & 16 ' $39.980 "$ & 1.8 & $\sim 2.1$ & yes \\
\hline $3 \mathrm{C} 454.3$ & $22^{h}$ & $53^{m}$ & $57.748^{s}$ & $16^{d}$ & $08^{\prime} 53.561 "$ & - & $\sim 9.5$ & yes \\
\hline
\end{tabular}

Notes: ${ }^{a}$ Estimated HBA fluxes are taken from the WENSS survey (Rengelink et al. 1997).

${ }^{b}$ The LOFAR flux at $225 \mathrm{MHz}$ is the mean of the preliminary fluxes at this frequency from LOFAR Cycle 1 observations. ${ }^{\star}$ Caveat: Please keep in mind that LOFAR flux calibration is still preliminary and the quality of these measurements has not been assessed yet.

(Rickett et al. 1984), which, if intrinsic to the sources, would be in excess of $10^{18} \mathrm{~K}$. It has been suggested that the variability at wavelengths longer than $6 \mathrm{~cm}$ is due to a combination of scintillation and intrinsic variability, with the former becoming increasingly dominant with increasing wavelength. Disentangling the two requires analysis of the frequency dependence of the variability, and can be aided by the identification of the annual cycles in the variations that would arise from scintillation. Monitoring a sample of blazars over a wide range of radio frequencies allows us to distinguish the effects of intrinsic variability from extrinsic effects such as interplanetary scintillation and to quantify the importance of the latter as a function of frequency. In conjunction with regular VLBI monitoring (e.g., the MOJAVE project, Lister et al. (2009)), this can improve our understanding of the nature of the extreme variability and therefore the structure and dynamics of the parsec-scale jets in blazars (e.g., Marscher et al. (2010)). To this end, we are carrying out the first ever metre-wavelength monitoring of a sample of five blazars on a fortnightly basis since February 2013, as part of the LOFAR Transients Key Science Project work (Fender et al. 2006).

Our targets (see Table 1) are selected to be representative of the blazar population, sampling sources with synchrotron peaks all the way from the infrared (e.g., AO $0235+164$ ) to the ultraviolet or X-ray bands (e.g., Mrk 501). All are being monitored at $\mathrm{cm} / \mathrm{mm}$ wavelengths, e.g. with the VLBA within the MOJAVE project and the OVRO 40-m telescope at $2 \mathrm{~cm}$ (Richards et al. 2011). Snapshot observations with the full available bandwidth of the High Band Antennas (i.e., $48 \mathrm{MHz}$ centred at $226 \mathrm{MHz}$ ) give constraints on fluxes that are dominated by calibration uncertainties, and certainly good enough to track the observed order-of-magnitude variability seen at $\mathrm{GHz}$ frequencies. Moreover, the cadence (fortnightly) matches that of $\mathrm{GHz}$ monitoring.

So far, LOFAR light curves are revealing a quite smooth behaviour; however, some of them show trends on time-scales of months which we believe to be real and could only be detected with a long-term monitoring campaign such as ours. On-going monitoring will allow us to investigate it deeper.

\section{References}

Fender, R. P., Wijers, R. A. M. J., Stappers, B., et al. 2006, PoS(MQW6)104

Lister, M. L., Cohen, M. H., Homan, D. C., et al. 2009, AJ, 138, 1874

Marscher, A. P., Jorstad, S. G., Larionov, V. M., et al. 2010, ApJ (Letters), 710, L126

Rengelink, R. B., Tang, Y., de Bruyn, A. G., et al. 1997, A\&SAS, 124, 259

Richards, J. L., Max-Moerbeck, W., Pavlidou, V., et al. 2011, ApJS, 194, 29

Rickett, B. J., Coles, W. A., \& Bourgois, G. 1984, A\&A, 134, 390

van Haarlem, M. P., Wise, M. W., Gunst, A. W., et al. 2013, A\&\&A, 556, A2 